\title{
Assessment of pulsed Doppler echocardiography in detection and quantification of aortic and mitral regurgitation ${ }^{\star}$
}

\author{
MIGUEL A QUINONES, JAMES B YOUNG, ALAN D WAGGONER, \\ MIODRAG C OSTOJIC, LAIR G T RIBEIRO, RICHARD R MILLER
}

From the Section of Cardiology, Department of Medicine, Baylor College of Medicine and The Methodist Hospital, Houston, Texas, USA

SUMMARY Pulsed Doppler echocardiography was employed to detect disturbed or turbulent flow diagnostic of aortic or mitral regurgitation. Sensitivity, specificity, diagnostic accuracy, and predictive value were assessed by the independent interpretation and comparison of aortic root angiograms (91 patients) and left ventriculograms (94 patients) to the time interval histogram display of the pulsed Doppler. Sensitivity of Doppler in detecting mitral regurgitation was 94 per cent, with specificity 89 per cent, predictive value 81 per cent, and diagnostic accuracy 90 per cent ( 32 patients with, 62 without regurgitation). In aortic regurgitation, sensitivity was also 94 per cent, specificity 82 per cent, predictive value 94 per cent, and the diagnostic accuracy was 91 per cent (69 patients with, 22 without aortic regurgitation). Additionally, no Doppler evidence of mitral or aortic regurgitation was present in 20 normal subjects. The aetiology of left-sided valvular regurgitation varied widely, with prosthetic valvular insufficiency being the cause of mitral and aortic regurgitation in seven and 10 patients, respectively. Sixteen of $17(94 \%)$ paraprosthetic leaks were correctly identified by pulsed Doppler. In patients with aortic regurgitation the flow-velocity curve recorded in the ascending aorta frequently showed a negative (or reversed) diastolic component, the magnitude of which (expressed as percentage negative area) correlated significantly with angiographic severity of regurgitation. Thus, pulsed Doppler echocardiography is a highly accurate and objective non-invasive technique for detecting mitral and aortic regurgitation. In aortic regurgitation, estimation of severity is possible from inspection of the Doppler ascending aortic flow velocity curve.

Pulsed Doppler echocardiography is a new noninvasive technique that has proved useful in the detection of valvular regurgitation or stenosis ${ }^{1-3}$ as well as structural abnormalities such as atrial or ventricular septal defects. ${ }^{4}$ The addition of pulsed Doppler studies to routine M-mode echocardiography enhances the likelihood of localising the source of cardiac murmurs and in certain instances quantifying the severity of valvular dysfunction. In this regard, we have recently shown ${ }^{6}$ that analysis of the time interval histogram of the pulsed Doppler can be of use in discriminating between patients with severe aortic stenosis (aortic valve area less than $1.0 \mathrm{~cm}^{2}$ ) and those with less severe

* Supported in part by the American Heart Association, Texas Affiliate, research grant, and the National Heart, Lung and Blood Vessel Research and Demonstration Center, Baylor College of Medicine.

Received for publication 24 March 1980 stenosis (aortic valve area greater than $1.0 \mathrm{~cm}^{2}$ ). Additionally, we have been able to quantify the amount of tricuspid regurgitation and relate the extent of regurgitation to right ventricular haemodynamics. ${ }^{?}$

Earlier reports concerning the diagnostic accuracy of pulsed Doppler echocardiography in detecting left-sided valvular regurgitation have depended primarily on a subjective interpretation of the audio output from the instrument to identify the presence of systolic turbulence in the left atrium, a sign of mitral regurgitation, ${ }^{8}$ or diastolic turbulence in the left ventricular outflow tract, a sign of aortic regurgitation. ${ }^{3}$ The obvious need for a graphic display of the data has led to the development of a time interval histogram for displaying the changes in frequency of the ultrasound. ${ }^{9}$

The objectives of this investigation were to 
determine the specificity, sensitivity, predictive values, and diagnostic accuracy of the time interval histogram in detecting the mitral and aortic regurgitation. Additionally, an attempt was made to correlate measurements derived from the time interval histogram with the angiographic severity of valvular insufficiency.

\section{Materials and methods}

\section{TECHNICAL DESCRIPTIONS}

The principles of pulsed Doppler echocardiography have been previously discussed by Johnson et al. ${ }^{1}$ and Baker et al. ${ }^{2}$ and will be reviewed only briefly. The frequency of a reflected sound wave is altered when the reflecting target is in motion. When an ultrasound beam is directed toward smooth, non-turbulent or laminar blood flow, the reflected sound waves exhibit a fairly uniform shift in frequency or "Doppler shift". This shift may be graphically represented as either a positive or negative deflection depending on direction of flow toward or away from the transducer. The magnitude of the shift is related to both velocity of flow and the angle of incidence between flow and sound. When the ultrasound beam is directed at turbulent blood flow, the reflected sound waves demonstrate a wide and random shift in frequency because of the varying direction and velocity of blood cells in the turbulent area.
Pulsed Doppler echocardiograms were performed in this study using a commercially available EchoDoppler unit (advanced Technology Laboratory, Bellevue, Washington) that employs repetitive pulses of sound in the megaHertz range similar to traditional M-mode echocardiography. The advantage of pulsed Doppler over continuous wave Doppler is that the Doppler shifts produced by the velocity of blood flow can be recorded in localised intracardiac positions at variable depths by means of a range-gating system in conjunction with a standard M-mode echocardiogram, thereby allowing accurate placement of the sample volume within the cardiac chambers. An audio signal is generated, which is represented as tonal quality changes. Non-turbulent blood flow results in a narrow frequency band width producing a musical sound in contrast to turbulent flow which generates a wide band width pattern producing a harsh rasping sound.

In addition to the aural presentation, a permanent display of the Doppler shift is achieved using a time interval histogram. The time interval histogram plots the frequency shifts of the reflected ultrasound using a zero-crossing technique that has previously been described by Lorch et al. ${ }^{9}$ In brief, a pattern is derived by measuring the time between successive zero crossings of audible signal wave forms. A zero crossing is defined as the instant in time when the Doppler shift signal
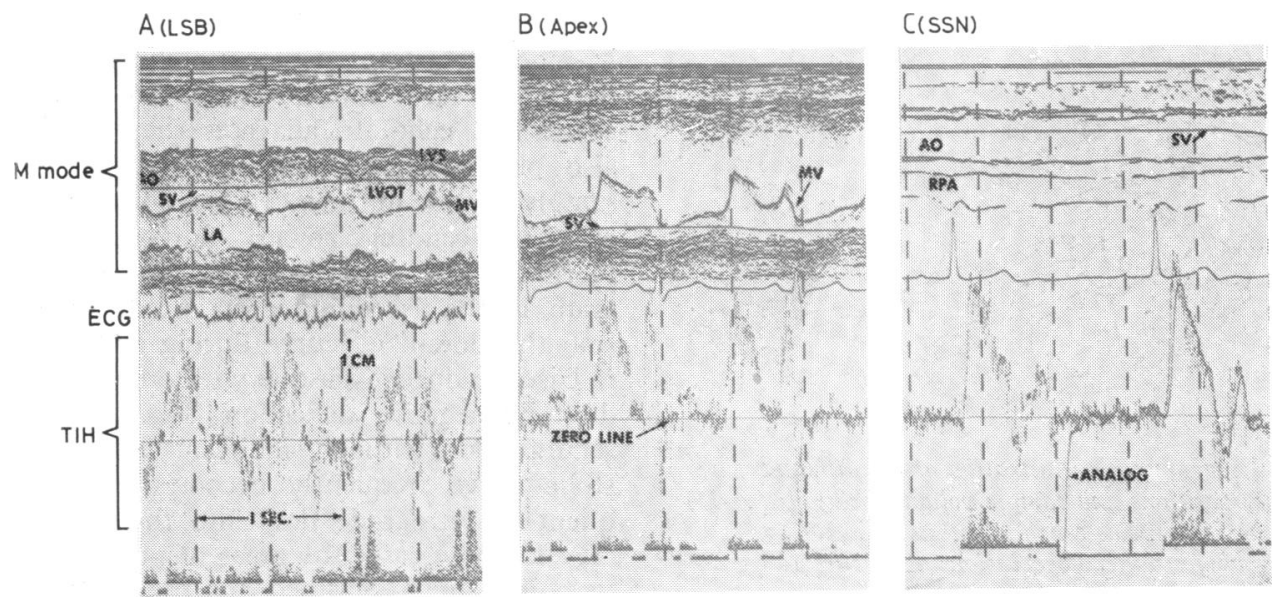

Fig. 1 Examples of a normal pulsed Doppler echocardiographic examination with a compressed M-mode on top and a time interval histogram (TIH) below. The sample volume is placed in the left ventricular outflow tract ( $L V O T$ ) when searching for aortic regurgitation (panel $A$ ) and posterior to the mitral valve (MV) when assessing mitral regurgitation (panel B). Panel $C$ illustrates a normal ascending aortic flow velocity curve. The transducer location is shown in each panel in parentheses. LSB, left sternal border; SSN, supra-sternal notch; $A O$, ascending aorta; IVS, interventricular septum; $L A$, left atrium; $R P A$, right pulmonary artery; ECG, electrocardiogram. 
passes through its zero intensity level. A series of dots are printed above (positive shift) or below (negative shift) a baseline point. Each dot represents a zero crossing event with the distance away from baseline reflecting the magnitude of the Doppler shift frequency (the greater the frequency shift, the shorter the zero crossing time interval). Smooth laminar flow produces a narrow cluster of dots while disturbed or turbulent flow produces a widely dispersed dot pattern. The ultimate display consists of the $\mathrm{M}$-mode echo with a superimposed line indicating the sample volume location, an electrocardiogram, and the time interval histogram (Fig. 1).

The technique employed in this study consisted of placing a $3.0 \mathrm{MHz}$ transducer along the left sternal border in a fashion similar to a routine M-mode echocardiographic study (Fig. 1 and 2). The mitral and aortic valves as well as the left ventricular outflow tract and left atrium were identified with the M-mode echocardiogram. Threshold and gain settings were adjusted with the sample volume placed in the left atrium. Threshold was set at the lowest level that allowed a slight background noise on the baseline (any further reduction in threshold would produce wide dispersion of the dots giving an appearance of turbulent flow even though no real turbulence would be detected by the aural analysis). Gain was set at the highest level that allowed only a slight background noise to be audible.

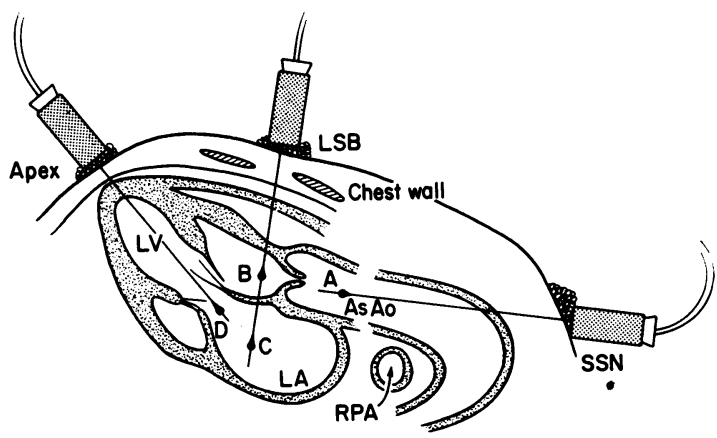

Fig. 2 Diagrammatic illustration of the different transducer positions and sample volume locations ( $A-D)$ used in this study. See text for details. As $A O$, ascending aorta : other abreviations as in Fig. 1.

Mitral regurgitation was sought from the left sternal border by placing the sample volume in the left atrium posterior to the anterior leaflet of the mitral valve or posterior to the aortic root while searching for systolic turbulence. In all cases the transducer was also placed at the cardiac apex with the patient in the left lateral recumbent position, the mitral valve was visualised, and the sample volume placed in the left atrium posterior to the anterior leaflet. When searching for turbulence, the operator relied on the audio output from the instrument while moving the sample volume around the left atrium making appropriate recordings when turbulence was heard. In all cases, however, the diagnosis of mitral regurgitation was ultimately based upon an objective analysis of the time interval histogram where turbulence was taken as a frequency dispersion greater than $10 \mathrm{~mm}$ (absolute measurement of dot distribution above and below the zero frequency shift baseline as shown in Fig. 3). When systolic turbulence was present, its maximal amplitude was measured in absolute millimetres from lines drawn outlining its upper and lower border (Fig. 3B). Measurements were averaged over the five cardiac cycles displaying widest frequency dispersion.

Aortic regurgitation was sought from the left sternal border by placing the sample volume in the left ventricular outflow tract (Fig. 4) just below the aortic valve, and searching for diastolic turbulence. As in mitral regurgitation, the diagnosis of aortic regurgitation was ultimately made from inspection of the time interval histogram. Special precaution in patients with left ventricular inflow obstruction (mitral stenosis or prosthetic mitral valves) was used to avoid confusion between diastolic turbulence produced by inflow through the mitral valve with that caused by aortic regurgitation. This potential source of error was prevented by sampling just below the aortic valve at a point where the M-mode echogram demonstrated the junction of the mitral valve anterior leaflet with the posterior aortic root echo. In all cases the transducer was also placed at the suprasternal notch with the sample volume in the ascending aorta for recording of the ascending aortic "flow-velocity" curve. Normally, the analogue of the time interval histogram in the ascending aorta produces a positive smooth "flow-like" curve during systole returning to baseline during diastole with only minimal early diastolic negative deflection (Fig. 1C). In aortic regurgitation without significant stenosis the diastolic curve frequently becomes negative consequent to retrograde flow into the ventricle (Fig. 4B). In' these cases the areas of positive and negative deflections outlined by the analogue curve were measured by planimetry in five consecutive cardiac cycles and the results averaged to compute an index of severity of aortic regurgitation:

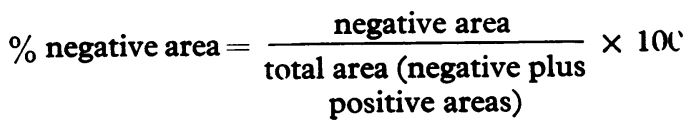



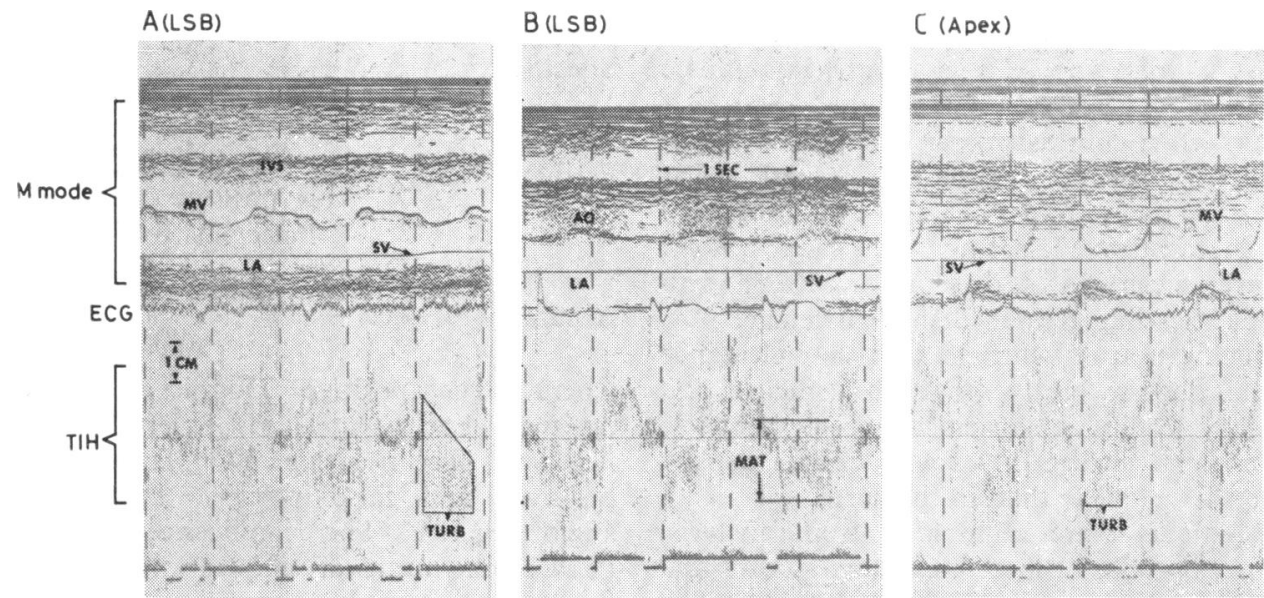

Fig. 3 Examples of systolic turbulence (TURB) indicative of mitral regurgitation with sample volume located posterior to mitral valve (panels $A$ and $C$ ) or in the left atrium (panel B); transducer position at left sternal border (panels $A$ and $B$ ) or cardiac apex (panel $C$ ). The maximal amplitude of turbulence (MAT) is measured as shown in panel B. See text for details. Abbreviations as in Fig. 1.

During a period of six months all patients who had a pulsed Doppler examination within five days of cardiac catheterisation were selected for analysis. The results from the Doppler time interval histogram were compared with the left ventricular or aortic root angiogram by independent observers. Left ventricular angiography was performed in all patients in a $30^{\circ}$ right anterior oblique position with the injection of 40 to $50 \mathrm{ml}$ Renografin-76 while the aortic root angiograms were performed in a $45^{\circ}$ left anterior oblique projection with injection of similar amounts of contrast material. Mitral regurgitation was diagnosed from the left ventriculogram when contrast material appeared in the left atrium during systole in the absence of ventricular or atrial ectopy. The severity of mitral regurgitation was judged qualitatively as $1+$ (rapid clearance of dye from the left atrium with each cycle), $2+$ (slower clearance of dye from left atrium but greater opacification of ventricle than atrium), or

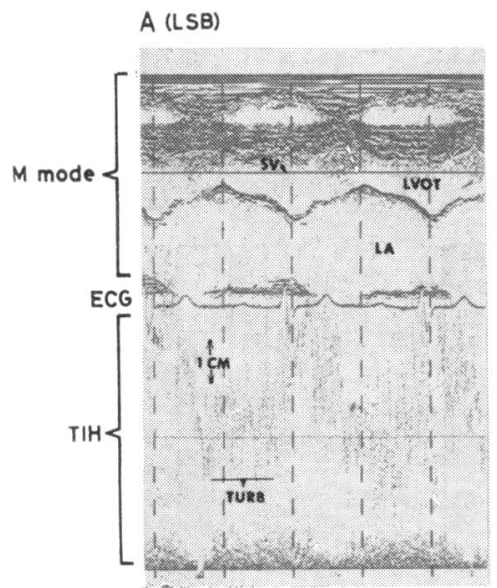

$B(S S N)$

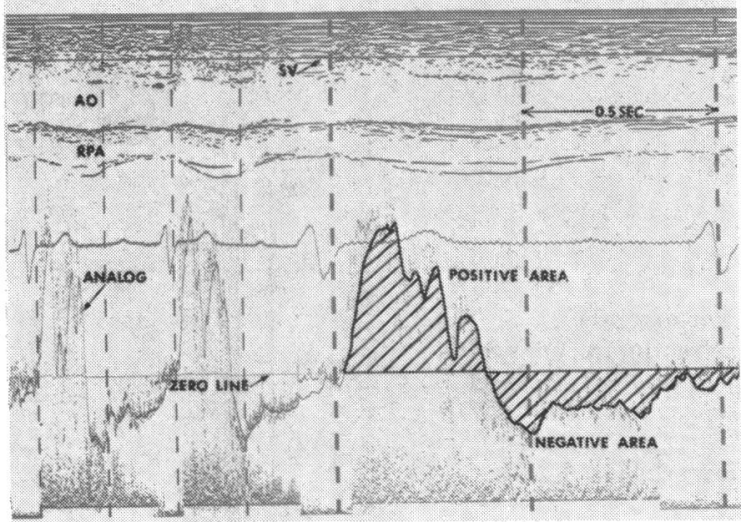

Fig. 4 Panel $A$ shows an example of diastolic turbulence (TURB) in the left ventricular outflow tract indicative of aortic regurgitation; transducer positioned at left sternal border. Panel $B$ illustrates an ascending aortic flow velocity curve from the same patient showing a characteristic diastolic negative deflection. Planimetry of the negative and positive areas allows assessment of severity of aortic regurgitation (see text). Abbreviations as in Fig. 1. 
3+ (left atrium filled with dye and appearing equal to or more dense than ventricle). Aortic regurgitation was diagnosed from the aortic root angiogram when contrast material appeared in the left ventricle during diastole; its severity was judged qualitatively from $1+$ to $4+: 1+$, minimal dye in the ventricle clearing with each systole; $2+$, slower clearance of dye from ventricle with aortic opacification greater than ventricular; $3+$, rapid opacification of ventricle with slow clearance and equal density of dye in both ventricle and aorta; $4+$, complete opacification of ventricle within first two cardiac cycles with greater density of dye in the ventricle than in the aorta.

Four patients were eliminated from analysis, two for suboptimal echocardiographic studies and two for suboptimal quality of the angiograms. The decision to eliminate these patients was made without knowledge of the results of the angiogram in the first two or the Doppler examination in the second two cases. A total of 94 studies was available for comparison with angiographic detection of mitral regurgitation and 91 for comparison with angiographic detection of aortic regurgitation. In addition, 20 normal subjects without evidence of organic heart disease were studied by pulsed Doppler echocardiography to establish specificity for mitral and aortic regurgitation in a normal population. The sensitivity, specificity, predictive value, and accuracy of the pulsed Doppler echocardiogram were calculated as follows:

Sensitivity $=\frac{\text { true positive }}{\text { true positive plus false negatives }}$
Specificity

$$
=\frac{\text { true negative }}{\text { true negative plus false positives }}
$$

Predictive value $=\frac{\text { true positive (or negative) }}{\text { true positive (or negative) plus }}$ false positive (or negative)

Accuracy $=\frac{\text { true positives plus true negatives }}{\text { all subjects }}$

where, true positive $=$ Doppler positive, angio positive; true negative $=$ Doppler negative, angio negative; false negative $=$ Doppler negative, angio positive; and false positive $=$ Doppler positive, angio negative. The significance of a difference between the means of samples was computed using Student's $t$ test with significance established at a $\mathrm{p}<0.05$ level.

\section{Results}

\section{MITRAL REGURGITATION}

Fig. 3 shows examples of mitral regurgitation detected from either left sternal border or apical approaches. Fig. 5 illustrates the sensitivity, specificity, and predictive values of Doppler in detecting mitral regurgitation. Angiographic evidence of mitral regurgitation was present in 32 out of 94 patients. Table 1 lists the aetiologies of mitral regurgitation in these patients. The pulsed Doppler time interval histogram detected mitral regurgitation in 30 of the 32 patients ( $94 \%$ sensitivity). Mitral regurgitation was absent by Doppler in 55 out of 62 patients without angiographic evidence of
Fig. 5 Bar diagram

illustrating sensitivity, specificity, and predictive values of pulsed Doppler echocardiography (PDE) in mitral regurgitation (MR). Angio, angiography.

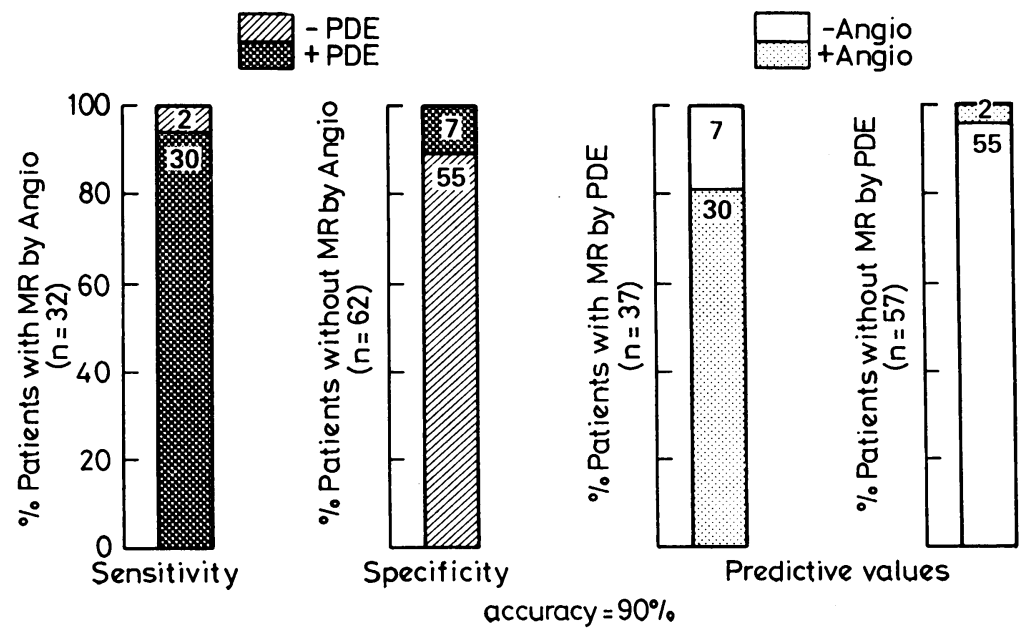


regurgitation $(89 \%$ specificity). The predictive values for a positive and a negative Doppler examination were 85 per cent and 96 per cent, respectively, while the overall diagnostic accuracy was 90 per cent. Both false negative examinations occurred in patients with minimal $(1+)$ angiographic mitral regurgitation. The seven false positive Doppler studies consisted of four patients with cardiomyopathy, and one each with aortic stenosis and left ventricular failure, mitral valve prolapse, and ventricular septal defect. None of the 20 normal subjects had evidence of mitral regurgitation by Doppler.

Table 1 Aetiology of mitral regurgitation in 32 patients

\begin{tabular}{lll}
\hline Aetiology & Angio MR & $P D E M R$ \\
\hline Rheumatic & 13 & 12 \\
Prosthetic valve leak & 7 & 6 \\
Myxomatous valve & 5 & 5 \\
Cardiomyopathy & 3 & 3 \\
Papillary muscle dysfunction & 3 & 3 \\
Congenital defect & 1 & 1 \\
\hline Total & 32 & 30 \\
\hline
\end{tabular}

Angio, angiographic; MR, mitral regurgitation; PDE, pulsed Doppler echocardiography.

Mitral regurgitation was detected from a single location in 19 patients and from multiple locations in 11 patients. In patients having regurgitation noted from a single location, turbulence was detected only in the left atrium in two, behind the mitral valve with the transducer at the left sternal border in eight, and behind the mitral valve with the transducer placed at the apex in nine. Turbu- lence was located in early systole in 10 patients, mid or late systole in six, and was holosystolic in 14. No correlation ( $p>0.05)$ was observed between duration or location of turbulence and angiographic severity of mitral regurgitation.

The maximum amplitude of systolic turbulence was $15.9 \pm 1.0 \mathrm{~mm}$ (mean \pm standard error), $18.9 \pm$ $1.9 \mathrm{~mm}$, and $21.8 \pm 1.4 \mathrm{~mm}$ in patients with $1+$, $2+$, and $3+$ mitral regurgitation, respectively. While a tendency for greater amplitude of turbulence to occur with increasing severity of mitral regurgitation was suggested, the overlap between individual values was such that only the comparison of $1+$ versus $3+(15.9$ versus $21.8 \mathrm{~mm})$ achieved statistical significance at the 0.05 level.

\section{AORTIC REGURGITATION}

Fig. 4A illustrates an example of aortic regurgitation detected with the sample volume in the left ventricular outflow tract. Fig. 6 illustrates the sensitivity, specificity, and predictive values of pulsed Doppler in detecting aortic regurgitation. Angiographic evidence of aortic regurgitation was present in 69 out of 91 patients; the time interval histogram of the pulsed Doppler was positive in 65 out of 69 patients ( $94 \%$ sensitivity). Aortic regurgitation was absent by Doppler in 18 out of 22 patients without angiographic evidence of aortic regurgitation ( $82 \%$ specificity). The predictive value for a positive and negative Doppler examination was 94 per cent and 82 per cent, respectively; the overall diagnostic accuracy was 91 per cent. The different aetiologies of aortic regurgitation are listed in Table 2.

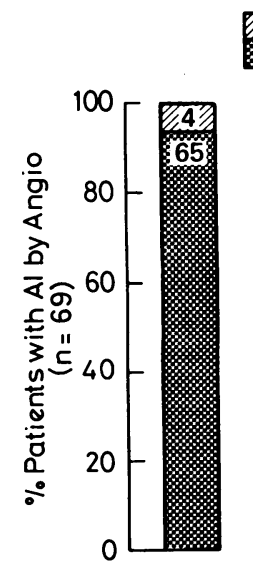

Sensitivity
- PDE
+ PDE

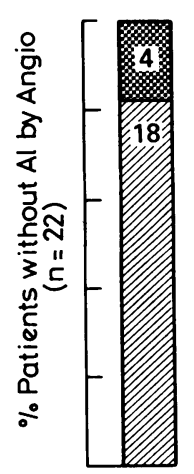

Specificity

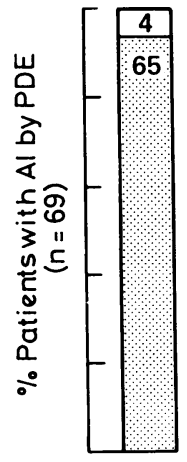

- Angio
+ Angio

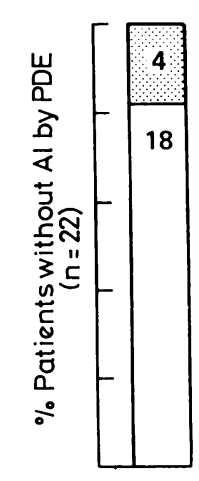

Predictive values
Fig. 6 Bar diagram illustrating sensitivity, specificity, and predictive values of pulsed Doppler echocardiography (PDE) in aortic insufficiency ( $A I)$. Angio, angiography. 
The four false negative Doppler examinations occurred in patients with minimal $(1+)$ angiographic aortic regurgitation. The four false positive Doppler studies consisted of three patients with rheumatic heart disease and evidence of aortic valve thickening by $M$-mode echocardiography, and one patient with a membranous ventricular septal defect, severe pulmonary hypertension, and significant right-to-left shunting. None of the 20 normal subjects had evidence of aortic regurgitation by Doppler.

Neither the duration nor the amplitude of diastolic turbulence in the left ventricular outflow tract correlated with the angiographic severity of aortic regurgitation. In contrast, the analogue of the time interval histogram "flow-velocity" curve recorded in the ascending aorta with the transducer in the suprasternal notch frequently demonstrated a negative diastolic component (Fig. 4B). The magnitude of negative deflection, measured as the percentage negative area in 54 of the 69 patients with aortic regurgitation, was found to relate significantly $(p<0.05)$ with the qualitative angiographic assessment of severity of regurgitation. As shown in Fig. 7, the percentage negative area was $8.9 \pm 2.9$ (mean $\pm S E M$ ) for patients with $1+$, $23 \cdot 6 \pm 4 \cdot 2$ for $2+, 35 \cdot 7 \pm 5$ for $3+$, and $50 \cdot 2 \pm 6 \cdot 5$ for $4+$ aortic regurgitation. A 30 per cent or less negative area was noted in 26 out of $31(84 \%)$ patients with $2+$ or less aortic regurgitation, while a negative area greater than 30 per cent was present in 17 out of $23(74 \%)$ patients with $3+$ or more regurgitation. Thus, using a less or greater than 30 per cent negative deflection to discriminate between mild $(\leqslant 2+)$ and severe $(\geqslant 3+)$ aortic regurgitation, the predictive value of the pulsed Doppler was 81 and 77 per cent, respectively, with a diagnostic accuracy of 80 per cent.

\section{Discussion}

Pulsed Doppler echocardiography allows assessment of intracardiac and aortic flow-velocity

Table 2 Aetiology of aortic regurgitation in 69 patients

\begin{tabular}{lll}
\hline Aetiology & Angio AR & PDE AR \\
\hline Rheumatic & 36 & 32 \\
Calcific & 12 & 12 \\
Prosthetic valve leak & 10 & 10 \\
Dilated aortic root & 6 & 6 \\
Endocarditis & 2 & 2 \\
Congenital defect & 2 & 2 \\
Myxomatous valve & 1 & 1 \\
\hline Total & 69 & 65 \\
\hline
\end{tabular}

AR, aortic regurgitation; others as in Table 1.

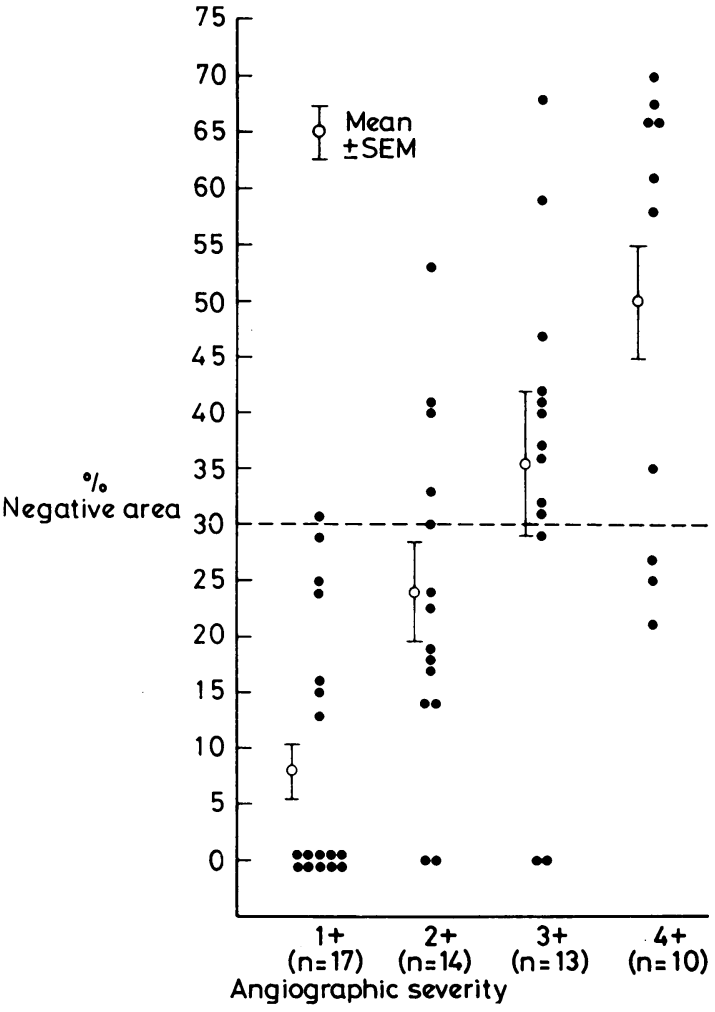

Fig. 7 Scatter diagram illustrating the percentage negative area of the ascending aortic flow velocity curve in patients with aortic regurgitation when compared with angiographic severity.

patterns not provided by standard $\mathrm{M}$-mode echocardiography. The recent development of a graphic display of the frequency shifts of the reflected sound waves has made it possible to record and evaluate objectively flow-velocity patterns, even though an accurate measurement of flow velocity is still not possible with the commercially available system. Despite this limitation, the results of this investigation demonstrate a high $(90 \%$ and $91 \%)$ accuracy for this technique in detecting both mitral and aortic valvular regurgitation when angiography was used as the standard for comparison. The sensitivity for detection of either lesion was 94 per cent with all of the false negative cases having minimal regurgitation at angiography. The specificity for mitral regurgitation was 89 per cent, with six of the seven false positive patients having either left ventricular dilatation with failure (five patients) or mitral valve prolapse (one patient), conditions in which mitral regurgitation may be present inconsistently. 
The specificity for aortic regurgitation was 82 per cent. Three of the four false positive cases had significant aortic valve thickening by echocardiography, with varying degrees of stenosis observed during cardiac catheterisation. The fourth false positive case of aortic regurgitation had a membranous ventricular septal defect with pulmonary hypertension and a significant right-to-left shunt; presumably the diastolic turbulence detected in the left ventricular outflow tract originated from diastolic right-to-left shunting through the septal defect since the diastolic filling pressures in the right ventricle exceeded that in the left ( 35 versus $8 \mathrm{mmHg}$ ). None of the 20 normal subjects tested had Doppler evidence of mitral regurgitation or aortic regurgitation. If these subjects are considered as true negatives, the specificity of the technique for mitral and aortic regurgitation becomes 91 and 90 per cent, with an accuracy of 92 and 93 per cent, respectively. These results are comparable to previous reports on detection of mitral and aortic regurgitation by pulsed Doppler echocardiography. ${ }^{38}$ The previous investigations, however, were solely dependent on the subjective interpretation of the audio output from the instrument by the technician performing the study while the present study relied on an objective interpretation of the time interval histogram.

Although the diagnosis of mitral or aortic regurgitation in this investigation was made from an objective inspection of the time interval histogram, the technical skills of the operator remain an important factor in obtaining an optimal study. Detection of mitral regurgitation is a clear example of this. Mitral regurgitation was detected from a single location in 19 out of 30 patients, the left sternal border approach being no more sensitive than the apex (eight of 19 versus nine of 19); in the remaining 11 patients mitral regurgitation was detected from multiple locations. Thus, accurate recognition of left-sided valvular regurgitation at the time of the procedure requires use of a standardised search procedure with placement of the sample volume visually guided by the compressed $M$-mode and aurally directed by the valve and flow sounds indigenous to the various chambers. In addition, appropriate recordings must be supervised carefully by the technicians performing the study to ensure accurate reproduction of the aural findings.

A potential limitation of the pulsed Doppler technique may occur when clinical situations demand examination with the sample volume located a long distance from the transducer. This problem may be manifest when mitral regurgitation is sought from the cardiac apex. As Fig. 2 indicates, the sample volume is near the distal limits of the range-gate when placed in the left atrium or immediately posterior to the mitral valve with the transducer at the apex. At these deeper sites within the heart, the pulse repetition frequency (range 3500 to 10000 pulses per second) must be diminished to avoid ambiguous echoes, the net result being an increase in the minimum Doppler shift that can be detected. Theoretically, this might decrease the sensitivity for detection of mitral regurgitation since milder cases may not create sufficiently large Doppler shifts to be recognised. Despite this potential limitation, the sensitivity of pulsed Doppler for mitral regurgitation in this study was 94 per cent and, interestingly, this lesion was detected solely through the apical approach in nine patients.

The aetiological causes of mitral and aortic regurgitation in the present study were multiple and included the entire spectrum of valvular heart disease seen in adult cardiology. Both structural lesions (such as rheumatic, myxomatous, and calcific disease) as well as functional disturbances (such as left ventricular dilatation with papillary muscle dysfunction or ascending aortic aneurysms with loss of aortic valve support) were frequently observed. The accuracy of pulsed Doppler in detecting regurgitation was similar in each of these entities. Importantly, 17 patients had prosthetic valves with paravalvular leaks, including seven mitral and 10 aortic prosthetic valves. Of these 17 patients, 16 or 94 per cent were accurately detected by pulsed Doppler when compared with angiography; the one false negative consisted of a patient with a mitral prosthesis and minimal regurgitation. Accurate assessment of patients with valvular prostheses represents an important contribution of pulsed Doppler echocardiography to the clinical evaluation of these patients since paraprosthetic leaks are frequently difficult to confirm by either physical examination or by standard $M$-mode echocardiography.

Optimal determination of the severity of valvular regurgitation by Doppler analysis requires quantification of regurgitant flow. The present instrumentation, however, does not afford precise measurement of flow or velocity of flow. Nevertheless, if the angle between sound and blood flow remains constant, changes in the area under the "flow-velocity" curve should reflect changes in flow. Using this basic assumption, a comparison was made between the degree of reverse, or negative, diastolic flow in the ascending aorta (expressed as per cent negative area) and a qualitative angiographic assessment of the severity of aortic regurgitation. Using 30 per cent negative area as the 
separation point, this simple measurement yielded an 81 per cent and 77 per cent predictive value for differentiating mild $(<2+)$ versus severe $(\geqslant 3+)$ aortic regurgitation, respectively (Fig. 6). Angiographic measurements of regurgitant fractions were not made in these patients. It is conceivable that such a precise assessment of degree of regurgitation might even relate to the percentage negative area by. Doppler better than the qualitative assessment.

Determination of severity of aortic regurgitation echocardiographically has relied primarily on evaluating the degree of the left ventricular dilatation and the "volume overload" pattern of wall motion, both of which are haemodynamic consequences rather than a true measurement of regurgitation; both are also dependent upon obtaining a high quality echocardiographic view of the left ventricle. Our experience has been that a pulsed Doppler ascending aorta flow-velocity curve can be recorded easily in the vast majority of patients regardless of the difficulty in obtaining an adequate image by standard $\mathrm{M}$-mode techniques. The results presented herein suggest that analysis of this curve provides a direct index of aortic regurgitation.

A rough correlation was observed in the present study between the qualitative angiographic assessment of severity of mitral regurgitation and the achieved maximal amplitude of turbulence detected be Doppler. Statistical significance $(p<0.05)$, however, was achieved only for the comparison between $1+$ and $3+$ mitral regurgitation, and thus the application of this Doppler measurement to individual patients with mitral regurgitation is very limited.

Future improvements in the instrumentation and signal processing of the data will further enhance the ability of this new non-invasive technique to detect and quantify valvular regurgitation. The results of this investigation, however, indicate that in its present state, pulsed Doppler echo- cardiography has become a useful clinical adjunct to the non-invasive evaluation of patients suspected of having left-sided valvular regurgitation.

\section{References}

1 Johnson SL, Baker DW, Lute RA, Dodge HT. Doppler echocardiography; the localisation of cardiac murmurs. Circulation 1973; 48: 810-22.

2 Baker DW, Rubenstein SA, Lorch GS. Pulsed Doppler echocardiography: principles and applications. Am F Med 1977; 63: 69-80.

3 Ward JM, Baker DW, Rubenstein SA, Johnson SL. Detection of aortic insufficiency by pulse Doppler echocardiography. $\mathcal{F C U} 1977$; 5: 5-10.

4 Goldberg SJ, Areias JC, Spitaels SEC, de Villeneuve VH. Use of time interval histographic output from echo-Doppler to detect left-to-right atrial shunts. Circulation 1978; 58: 147-52.

5 Stevenson JG, Kawabori I, Dooley T, Guntheroth WG. Diagnosis of ventricular septal defect by pulsed Doppler echocardiography: sensitivity, specificity and limitations. Circulation 1978; 58: 322-6.

6 Young JB, Quinones MA, Waggoner AD, Miller RR. Diagnosis and quantification of aortic stenosis by pulsed Doppler echocardiography (abstract). Circulation 1978; 58, suppl II : 42.

7 Waggoner AD, Quinones MA, Verani MS, Miller RR. Pulsed Doppler echocardiographic detection of tricuspid insufficiency: diagnostic sensitivity and correlation with right ventricular hemodynamics (abstract). Circulation 1978; 58, suppl II : 41.

8 Johnson SL, Baker DW, Lute RA, Murrary JA. Detection of mitral regurgitation by Doppler echocardiography (abstract). Am f Cardiol 1974; 33: 146.

9 Lorch G, Rubenstein S, Baker D, Dooley T, Dodge H. Doppler echocardiography: use of a graphical display system. Circulation 1977; 56: 576-85.

Requests for reprints to Dr Miguel A Quinones, Section of Cardiology, Department of Medicine, Baylor College of Medicine, The Methodist Hospital MS No F1001, 6565 Fannin Street, Houston, Texas 77030, USA. 